\title{
Satisfacción de los pacientes que asisten a consulta externa, Centro de Salud San Miguel de Tuta Boyacá 2019
}

\author{
María A Varela-Suárez¹, Angie M Guzmán-Garay², Yury M Arenas
}

\section{RESUMEN}

Introducción. La calidad de la atención en salud se determina a través de la satisfacción de los usuarios, con base en sus necesidades y asociada a elementos tangibles, de seguridad, fiabilidad, sensibilidad y empatía, con respecto a los servicios y al personal de salud.

Objetivo. Identificar la satisfacción de los pacientes que asisten al servicio de consulta externa en el ESE Centro de Salud San Miguel de Tuta, Boyacá.

Materiales y métodos. Se desarrolló un estudio cuantitativo, descriptivo, con diseño transversal y una muestra de 76 pacientes, obtenida mediante un muestreo probabilístico a través del software EPI INFO.

Resultados. De acuerdo con el análisis de las dimensiones del estudio, se obtuvieron resultados de mejor satisfacción, anteponiendo algunas atenciones; asimismo, desde la perspectiva de los usuarios que acuden al Centro de Salud San Miguel del municipio de Tuta, se observó que los usuarios están muy de acuerdo con las dimensiones de elementos tangibles y empatía y en lo concerniente a las dimensiones de fiabilidad, sensibilidad y seguridad.

Conclusiones. A partir del estudio, se evidencia satisfacción por parte de los usuarios en el servicio de consulta externa, lo cual favorece sus preferencias hacia las consultas de control prenatal y permite evidenciar la buena atención, las expectativas y percepciones de los usuarios.

Palabras clave: garantía de la calidad de atención de salud, derivación y consulta, satisfacción del paciente, empatía.

\footnotetext{
${ }^{1}$ Universidad de Boyacá. Tunja. Boyacá. Colombia

2 ESE Centro de Salud Nuestra Señora de la Paz. Quipama. Boyacá. Colombia
}

Correspondencia: Yury Marcela Arenas. Dirección: Diagonal 67-1-09 Barrio Muiscas, Tunja Boyacá, Colombia. Teléfono: (321)3965068.

Correo electrónico: ymarenas@uniboyaca.edu.co.

Citar este artículo así:

Varela-Suárez MA, Guzmán-Garay AM, Arenas YM. Satisfacción de los pacientes que asisten a consulta externa, Centro de Salud San Miguel de Tuta Boyacá 2019. Revista Investig Salud Univ Boyacá. 2020;7(1): 87-101. doi: https://doi.org/10.24267/23897325.425 


\title{
Satisfaction of patients attending an outpatient clinic, San Miguel Tuta Boyacá health center 2019.
}

\begin{abstract}
Introduction. The quality of health care is determined through the satisfaction of users based on their needs and associated with tangible elements, security, reliability, sensitivity and empathy of services and health personnel.
\end{abstract}

Objective. Identify the satisfaction of the patients who attend the outpatient service at the ESE San Miguel de Tuta Boyacá Health Center.

Materials and methods. A quantitative, descriptive, cross-sectional study was developed, the sample of 76 patients was obtained by probabilistic sampling through EPI INFO software.

Results. According to the analysis of the study dimensions, results of better satisfaction were obtained, putting some attention first; from the perspective of the users who come to the San Miguel Health Center in the municipality of Tuta, it was obtained that the users agree very much with the dimensions of tangible elements and empathy and in relation to the dimensions of reliability, sensitivity and security.

Conclusions. From the study, there is evidence of satisfaction on the part of the users in the outpatient service, favoring their preferences towards prenatal care consultations, evidencing against the good care, expectations and perceptions of the users.

Keywords: quality assurance of health care, referral and consultation, patient satisfaction, empathy. 
Satisfação dos pacientes atendidos no serviço ambulatório, do Centro de Saúde San Miguel de Tuta Boyacá 2019

\section{RESUMO}

Introdução. A qualidade da atenção em saúde é determinada pela satisfação dos usuários, com base em suas necessidades e são associadas a elementos tangíveis de segurança, confiabilidade, sensibilidade e empatia relacionadas aos serviços e ao pessoal de saúde.

Objetivo. Identificar a satisfação dos pacientes que comparecem ao serviço ambulatório na ESE Centro de Saúde San Miguel de Tuta, Boyacá.

Materiais e métodos. Foi realizado um estudo quantitativo, descritivo, com delineamento transversal e com uma amostra de 76 pacientes, obtidos por meio de uma amostragem probabilística no software EPI INFO.

Resultados. De acordo com a análise das dimensões do estudo, foram obtidos resultados de melhor satisfação, colocando algum tipo de atenção em primeiro lugar; da mesma forma, na perspectiva dos usuários que assistem ao Centro de Saúde San Miguel, no município de Tuta, observou-se que os usuários concordam com as dimensões dos elementos tangíveis e da empatia e com as dimensões da confiabilidade, sensibilidade e segurança.

Conclusões. A partir do estudo, a satisfação é evidenciada pelos usuários no serviço ambulatorial, o que favorece suas preferências em relação à consulta pré-natal e permite evidenciar bons cuidados, expectativas e percepções dos usuários. 


\section{INTRODUCCIÓN}

La diversidad de cambios en la relación profesional de salud y paciente, ha presentado variaciones importantes en los últimos años, generado en algunos casos, inconformidades en ambas partes, o por el contrario, satisfacción del usuario. Ésta última, considerada como el principal factor que mide la calidad en la atención de salud y el máximo grado de complacencia que tiene el usuario basado en sus necesidades, teniendo en cuenta el cuidado integral, la atención oportuna, la seguridad del paciente y la calidad de la atención (1).

Para evaluar la calidad de servicio en salud, es necesario medir el grado de satisfacción del usuario, mediante la diferencia existente entre la expectativa y la percepción del mismo (2); de esta manera, es posible ayudar a los establecimientos de salud a evaluar hasta qué punto la cultura de seguridad de su organización transmite la importancia de la seguridad del paciente (3).

De acuerdo con la literatura, la satisfacción de los usuarios asistentes a consulta externa se asocia a elementos tangibles, de seguridad, fiabilidad, sensibilidad y empatía de los servicios y del personal de salud (4-7), así, estas investigaciones se orientan precisamente hacia la satisfacción del usuario, enfatizando en diferentes aspectos por mejorar, tales como el recurso físico y humano de la institución (8-11).
La forma en que la gestión del cuidado interviene en la calidad de la atención de salud genera la satisfacción del usuario, así como la atención y trato humano por el profesional de salud $(6,12)$, con lo cual se logra una adecuada comunicación entre usuarios y profesionales, lo que a su vez contribuye a transmitir la información de forma clara, vinculando a los pacientes en la toma de decisiones sobre la atención, la competencia profesional de los prestadores de los servicios de salud y las cualidades personales para interactuar con el usuario (13-15).

Finalmente, es importante mencionar que la calidad de los servicios de salud se ha analizado adoptando una metodología cuantitativa, encuestas a usuarios; forma en la cual, la mayoría de los cuestionarios que se utilizan, con excepción del SERVQUAL, carecen de marcos conceptuales sólidos (12). Debido a lo expuesto anteriormente, es recomendable para las instituciones de salud utilizar el modelo Service of Quality (SERVQUAL), ya que permite identificar los factores determinantes de la calidad del servicio y la percepción del usuario (16-18); por este motivo, la correcta adherencia a las guías de atención, y a los instrumentos que miden la calidad del servicio por parte del profesional de salud, es fundamental en el momento de brindar educación y recomendaciones a los usuarios en busca de disminuir los factores de riesgo y ofrecer un servicio con calidad. Por lo tanto, el propósito de este estudio 
es identificar la satisfacción de los pacientes que asisten al servicio de consulta externa en el ESE Centro de Salud San Miguel de Tuta Boyacá, año 2019.

\section{MATERIALES Y MÉTODOS}

En este estudio de tipo descriptivo, con cohorte transversal, se llevó a cabo un diseño de campo sobre la satisfacción de los pacientes que asisten al servicio de consulta externa, con base en datos de los usuarios que asistieron al servicio de consulta externa en la E.S.E Centro de Salud San Miguel de Tuta Boyacá; recolectados durante el mes de noviembre del 2019, teniendo como criterios de inclusión a todas las personas mayores de edad usuarias del servicio de consulta externa durante el segundo semestre del año 2019 y su aceptación para participar, excluyendo a los usuarios del servicio de urgencias. Asimismo, se empleó un muestreo probabilístico con una muestra de 76 usuarios, obtenidos con el software Epidat versión 7.2.3.1 del 28 junio 2019 del Centro para el Control y la Prevención de Enfermedades, desarrollado en Atlanta Georgia, bajo un nivel de confianza del $95 \%$ y una potencia: mínimo y máximo del $5 \%$.

El instrumento de recolección de información fue una encuesta del modelo SERVQUAL que consiste en un cuestionario con preguntas estandarizadas para la Medición de la Calidad del Servicio; se trata de una herramienta desarrollada por Valerie A. y colaboradores, implementada en los Estados Unidos y validada a América Latina por Michelsen Consulting. El Estudio de Validación concluyó en junio de 1992. El modelo SERVQUAL es una técnica de investigación comercial que permite realizar la medición de la calidad del servicio, conocer las expectativas de los clientes y la forma cómo ellos aprecian el servicio (3).

En la primera parte del instrumento se incluyó la información sociodemográfica del participante, como edad, sexo, nivel educativo y estrato; en la segunda parte se continuó con preguntas estandarizadas que valoraran las dimensiones, las cuales se dividen en 5, así: elementos tangibles, fiabilidad, sensibilidad, seguridad y empatía. Para las dimensiones de fiabilidad y empatía se diseñaron cinco preguntas respectivamente $y$, para las dimensiones de elementos tangibles, sensibilidad y seguridad, se diseñaron cuatro preguntas para cada una.

Los ítems incluían siete opciones de respuesta, en una escala tipo Likert (2), con las siguientes puntuaciones: valor de 1 si estaba nada de acuerdo (NA), 2 cuando estaba en desacuerdo (DD), 3 cuando estaba parcialmente en desacuerdo (PD), 4 cuando estaba regularmente de acuerdo (RA), 5 cuando estaba parcialmente de acuerdo (PA), 6 cuando estaba de acuerdo (DA) y 7 cuando estaba muy de acuerdo (MA). 
La investigación contó con la aprobación del comité de ética de la Universidad de Boyacá y autorización por parte de la gerencia administrativa de la Institución de salud E.S.E. Centro de Salud San Miguel municipio de Tuta, además, con el consentimiento informado por parte de cada uno de los participantes que conformaron la población de estudio.

\section{RESULTADOS DATOS SOCIODEMOGRÁFICOS}

Los datos sociodemográficos de la muestra son: edad, nivel educativo, sexo y estrato; de los cuales se pudo evidenciar que el $25 \%$ están entre las edades de 35-43 años, seguido del $24 \%$ que corresponde entre los 26-34 años, con mayor frecuencia de población femenina (64\%) en relación a la población masculina que corresponde al $36 \%$, el nivel de escolaridad representativo fue secundaria (49\%) y el de menor puntaje fue ninguno relacionado con el 3\% y con estrato socioeconómico del uno (58\%).

\section{DATOS DE CADA DIMENSIÓN}

Las dimensiones de la variable son elementos tangibles (tabla1), fiabilidad (tabla 2), sensibilidad (tabla 3), seguridad (tabla 4) y empatía (tabla5). Nada de acuerdo (NA), desacuerdo (DD), parcialmente en desacuerdo $(P D)$, regularmente de acuerdo (RA), parcialmente de acuerdo (PA), de acuerdo (DA) y muy de acuerdo (MA).

Tabla 1: Dimensión elementos tangibles establecida por los usuarios que asisten al servicio de consulta externa.

En la E.S.E Centro de Salud San Miguel, municipio de Tuta. Departamento de Boyacá, noviembre 2019

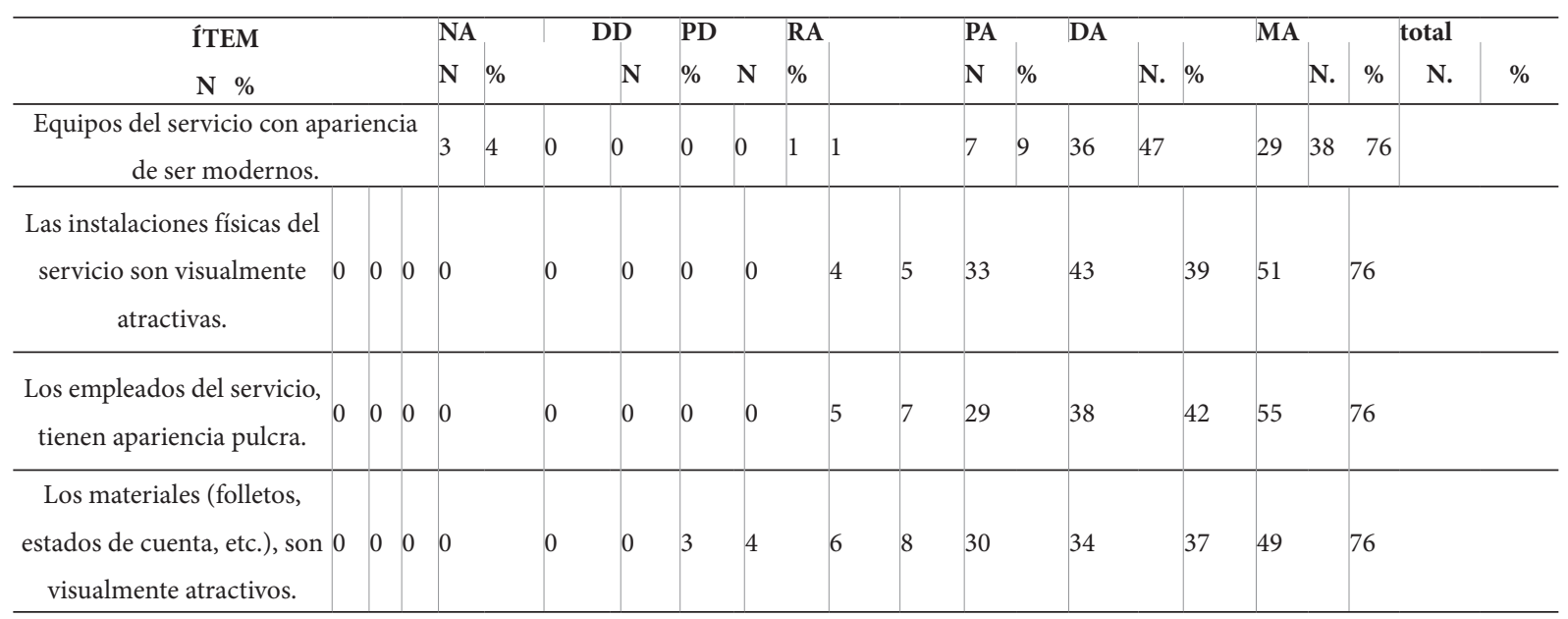

Fuente: datos obtenidos de la aplicación de la encuesta a la población objeto de estudio. Noviembre 2019 
En el ítem número 1 que evalúa los equipos del servicio, los usuarios encuestados manifiestan, con un $47 \%$, estar de acuerdo con que el servicio tiene equipos modernos y atractivos, frente al $1 \%$ que está regularmente de acuerdo con dicha afirmación. El ítem número 2 evalúa las instalaciones del servicio; en este, los usuarios encuestados manifiestan, con un $51 \%$, estar muy de acuerdo en que las instalaciones físicas del servicio de consulta externa son visualmente atractivas, frente al $5 \%$ que está parcialmente de acuerdo con dicha afirmación. El ítem numero 3 evalúa la apariencia de limpieza del personal de salud, los usuarios manifiestan, en un $55 \%$, que están muy de acuerdo con la apariencia de limpieza del personal, comparado con el $7 \%$ que está parcialmente de acuerdo con dicha afirmación. Por último, en el ítem número 4 referente a los materiales del servicio, los usuarios manifiestan, con un $49 \%$, estar de acuerdo con los materiales concernientes al servicio de consulta externa (folletos, estados de cuenta, etc.), en relación con el $4 \%$ que está regularmente de acuerdo.

Tabla 2: Dimensión fiabilidad establecida por los usuarios que asisten al servicio de consulta externa. En la E.S.E Centro de Salud San Miguel, municipio de Tuta. Departamento de Boyacá, noviembre 2019

\begin{tabular}{|c|c|c|c|c|c|c|c|c|c|c|c|c|c|c|c|c|}
\hline \multirow{2}{*}{ ÍTEM } & \multicolumn{3}{|c|}{ NA } & \multicolumn{2}{|c|}{ DD } & \multicolumn{2}{|c|}{ PD } & \multicolumn{2}{|r|}{ RA } & \multicolumn{2}{|r|}{ PA } & \multicolumn{2}{|r|}{ DA } & \multicolumn{2}{|c|}{ MA } & \multirow[t]{2}{*}{ total } \\
\hline & $\mathbf{N}$ & $\%$ & $\mathbf{N}$ & $\%$ & & $\mathbf{N}$ & $\%$ & $\mathbf{N}$ & $\%$ & N. & $\%$ & N. & $\%$ & N. & $\%$ & \\
\hline $\begin{array}{l}\text { Cuando en el servicio de consulta externa prometen } \\
\text { hacer algo en cierto tiempo, lo hacen. }\end{array}$ & 2 & 3 & 0 & 0 & & 1 & 1 & 2 & 3 & 8 & 11 & 40 & 53 & 23 & 30 & 76 \\
\hline $\begin{array}{l}\text { Cuando usted tiene un problema con consulta } \\
\text { externa, muestran un sincero interés en solucionarlo. }\end{array}$ & 1 & 1 & 0 & 0 & & 0 & 0 & 0 & 0 & 10 & 13 & 36 & 47 & 29 & 38 & 76 \\
\hline $\begin{array}{l}\text { En consulta externa realizan bien el } \\
\text { servicio a la primera. }\end{array}$ & 2 & 3 & 0 & 0 & & 0 & 0 & 0 & 0 & 5 & 7 & 35 & 46 & 34 & 45 & 76 \\
\hline $\begin{array}{l}\text { En consulta externa concluyen el servicio en el } \\
\text { tiempo prometido. }\end{array}$ & 3 & 4 & 0 & 0 & & 0 & 0 & 2 & 3 & 6 & 8 & 35 & 46 & 30 & 39 & 76 \\
\hline $\begin{array}{l}\text { En consulta externa insisten en mantener registros } \\
\text { exentos de de errores. }\end{array}$ & 1 & 1 & 0 & 0 & & 0 & 0 & 0 & 0 & 10 & 13 & 39 & 51 & 26 & 34 & 76 \\
\hline
\end{tabular}

Fuente: datos obtenidos de la aplicación de la encuesta a la población objeto de estudio. Noviembre 2019. 
El ítem número 5 evalúa cuando en el servicio de consulta externa prometen hacer algo en cierto tiempo, los usuarios manifiestan, con un $53 \%$, estar de acuerdo en que actúan en corto tiempo teniendo en cuenta las necesidades. El ítem número 6 evalúa interés en ayudar al usuario según su duda, ellos manifiestan, con un $47 \%$, estar de acuerdo en que el personal de salud muestra interés en resolver las dudas. El ítem número 7 evalúa si el personal realiza bien el servicio a la primera; en este caso, los usuarios manifiestan con un $46 \%$ que están de acuerdo con la atención del servicio. El ítem número 8 evalúa el tiempo óptimo durante la consulta; al respecto, los usuarios encuestados manifiestan, en un $46 \%$, estar de acuerdo con el tiempo establecido para el servicio, en relación con un pequeño porcentaje del $8 \%$ que está parcialmente de acuerdo con dicha afirmación; por último, en el ítem número 9 que evalúa la existencia de registros exentos de errores, los usuarios manifiestan, con un $51 \%$, que están de acuerdo.

En el ítem número 10 que evalúa la información brindada con precisión a los usuarios al final de la prestación del servicio, los usuarios manifiestan, con un $51 \%$, estar de acuerdo con el compromiso del profesional para brindar la información pertinente, con base en sus necesidades, en contraste con el $5 \%$ que está regularmente de acuerdo

Tabla 3: Dimensión sensibilidad establecida por los usuarios que asisten al servicio de consulta externa. En

la E.S.E Centro de Salud San Miguel, municipio de Tuta. Departamento de Boyacá, noviembre 2019

\begin{tabular}{|c|c|c|c|c|c|c|c|c|c|c|c|c|c|c|c|}
\hline \multirow{2}{*}{ ÍTEM } & \multicolumn{2}{|c|}{ NA } & \multicolumn{2}{|c|}{ DD } & \multicolumn{2}{|c|}{ PD } & \multicolumn{2}{|c|}{ RA } & \multicolumn{2}{|c|}{ PA } & \multicolumn{2}{|c|}{ DA } & \multicolumn{2}{|c|}{ MA } & \multirow[t]{2}{*}{ total } \\
\hline & N. & $\%$ & N. & $\%$ & N. & $\%$ & N. & $\%$ & N. & $\%$ & N. & $\%$ & $\mathbf{N}$ & $\%$ & \\
\hline $\begin{array}{l}\text { Los empleados de consulta externa, informan } \\
\text { con precisión a los clientes cuándo concluirá la } \\
\text { realización de un servicio. }\end{array}$ & 0 & 0 & 0 & 0 & 2 & 3 & 4 & 5 & 6 & 7 & 39 & 51 & 28 & 37 & 76 \\
\hline $\begin{array}{l}\text { Los empleados de consulta externa } \\
\text { le sirven con rapidez. }\end{array}$ & 0 & 0 & 0 & 0 & 0 & 0 & 3 & 4 & 3 & 4 & 49 & 64 & 21 & 28 & 76 \\
\hline $\begin{array}{l}\text { Los empleados de consulta externa, siempre } \\
\text { están dispuestos a ayudarles. }\end{array}$ & 2 & 3 & 0 & 0 & 0 & 0 & 1 & 1 & 6 & 8 & 41 & 54 & 26 & 34 & 76 \\
\hline $\begin{array}{l}\text { Los empleados de consulta externa nunca están } \\
\text { demasiado ocupados para responder } \\
\text { a sus preguntas. }\end{array}$ & 0 & 0 & 0 & 0 & 0 & 0 & 1 & 1 & 8 & 11 & 37 & 49 & 30 & 39 & 76 \\
\hline
\end{tabular}

Fuente: datos obtenidos de la aplicación de la encuesta a la población objeto de estudio. Noviembre 2019. 
con esta afirmación. El ítem número 11 evalúa la ayuda brindada por el personal del servicio; en este, el $64 \%$ de los usuarios está de acuerdo con la ayuda brindada por el personal, en relación con el $4 \%$ que está parcialmente de acuerdo. El ítem número 12 que evalúa la disponibilidad del personal para ayudar a los usuarios, arroja un $54 \%$ de acuerdo con disponibilidad por parte del personal, sin embargo, el $8 \%$ restante está parcialmente de acuerdo. En el ítem número 13 que evalúa si el personal nunca está demasiado ocupado para responder a sus preguntas, el $49 \%$ de los usuarios está de acuerdo con la respuesta dada por parte del personal de salud, comparado con el $11 \%$ que está parcialmente de acuerdo.
El ítem número 14 evalúa el comportamiento de los empleados de consulta externa, el $72 \%$ de los usuarios está de acuerdo con el comportamiento del personal de salud al momento de la atención, dado que transmite confianza. El ítem número 15 evalúa la seguridad del usuario al momento de las transacciones en consulta externa, el 55\% de los usuarios está muy de acuerdo con la seguridad del personal de salud frente a las transacciones, en relación con un pequeño porcentaje del $7 \%$ que está parcialmente de acuerdo. El ítem número 16 evalúa amabilidad por parte del personal de salud, los usuarios encuestados manifiestan, en un $50 \%$, que están muy de acuerdo con amabilidad que reciben por parte del personal de

Tabla 4: Dimensión seguridad establecida por los usuarios que asisten al servicio de consulta externa. En la E.S.E Centro de Salud San Miguel, municipio de Tuta. Departamento de Boyacá, noviembre 2019.

\begin{tabular}{|c|c|c|c|c|c|c|c|c|c|c|c|c|c|c|c|}
\hline \multirow{2}{*}{ ÍTEM } & \multicolumn{2}{|c|}{ NA } & \multicolumn{2}{|c|}{ DD } & \multicolumn{2}{|l|}{ PD } & \multicolumn{2}{|l|}{ RA } & \multicolumn{2}{|l|}{ PA } & \multicolumn{2}{|l|}{ DA } & \multicolumn{2}{|c|}{ MA } & \multirow[t]{2}{*}{ total } \\
\hline & N. & $\%$ & N. & $\%$ & N. & $\%$ & N. & $\%$ & N. & $\%$ & N. & $\%$ & N. & $\%$ & \\
\hline $\begin{array}{l}\text { El comportamiento de los empleados de } \\
\text { consulta externa, le transmite confianza. }\end{array}$ & 0 & 0 & 0 & 0 & 0 & 0 & 0 & 0 & 4 & 0 & 38 & 72 & 34 & 23 & 76 \\
\hline $\begin{array}{l}\text { Usted se siente seguro en sus transacciones } \\
\text { con consulta externa }\end{array}$ & 1 & 1 & 0 & 0 & 0 & 0 & 1 & 1 & 5 & 7 & 27 & 36 & 42 & 55 & 76 \\
\hline $\begin{array}{l}\text { Los empleados de consulta externa, son } \\
\text { siempre amables con usted. }\end{array}$ & 0 & 0 & 0 & 0 & 0 & 0 & 0 & 0 & 4 & 5 & 34 & 45 & 38 & 50 & 76 \\
\hline $\begin{array}{l}\text { Los empleados de consulta externa, tienen } \\
\text { conocimientos suficientes para responder a } \\
\text { las preguntas que les hace. }\end{array}$ & 0 & 0 & 0 & 0 & 0 & 0 & 0 & 0 & 5 & 6,6 & 36 & 47 & 37 & 49 & 76 \\
\hline
\end{tabular}

Fuente: datos obtenidos de la aplicación de la encuesta a la población objeto de estudio. Noviembre 2019. 
salud, sin embargo, el $5 \%$ está parcialmente de acuerdo. El ítem número 17 evalúa los conocimientos de capacidad de respuesta del personal de salud frente a las necesidades de los usuarios, donde el $49 \%$ de los usuarios está muy de acuerdo con la capacidad de respuesta por parte del personal del profesional de salud y el $7 \%$ restante está parcialmente de acuerdo.

El ítem número 18 evalúa la atención individualizada al momento de la atención, los usuarios encuestados manifiestan, con un $58 \%$, estar muy de acuerdo con la existencia de atención individualizada al momento de la consulta, en relación con el 3\% restante que está parcialmente de acuerdo con esta afirmación. El ítem número 19 evalúa la flexibilidad de horarios para dar la atención, donde el 53\% de los usuarios está muy de acuerdo con la flexibilidad de los horarios, frente al $5 \%$ que está parcialmente de acuerdo con dicha afirmación. El ítem número 20 evalúa la atención personal al momento de la consulta, el $49 \%$ de los usuarios está muy de acuerdo en la atención personal. El ítem numero 21 evalúa que en el servicio se preocupan por sus mejores intereses, el $43 \%$ de los usuarios está muy de acuerdo con que en el momento de la consulta se sienten tranquilos y seguros, en con el $3 \%$ restante que

Tabla 5: Dimensión empatía establecida por los usuarios que asisten al servicio de consulta externa. En la E.S.E Centro de Salud San Miguel, municipio de Tuta. Departamento de Boyacá noviembre 2019

\begin{tabular}{|c|c|c|c|c|c|c|c|c|c|c|c|c|c|c|c|}
\hline \multirow{2}{*}{ ÍTEM } & \multicolumn{2}{|c|}{ NA } & \multicolumn{2}{|c|}{ DD } & \multicolumn{2}{|c|}{ PD } & \multicolumn{2}{|c|}{ RA } & \multicolumn{2}{|c|}{ PA } & \multicolumn{2}{|c|}{ DA } & \multicolumn{2}{|c|}{ MA } & \multirow[t]{2}{*}{ total } \\
\hline & N. & $\%$ & N. & $\%$ & N. & $\%$ & N. & $\%$ & N. & $\%$ & N. & $\%$ & N. & $\%$ & \\
\hline $\begin{array}{c}\text { En consulta externa le dan una atención } \\
\text { individualizada. }\end{array}$ & 0 & 0 & 0 & 0 & 0 & 0 & 0 & 0 & 2 & 3 & 30 & 39 & 44 & 58 & 76 \\
\hline $\begin{array}{c}\text { En consulta externa tienen horarios de } \\
\text { trabajo convenientes para } \\
\text { todos sus clientes. }\end{array}$ & 0 & 0 & 0 & 0 & 0 & 0 & 0 & 0 & 4 & 5 & 32 & 42 & 40 & 53 & 76 \\
\hline $\begin{array}{l}\text { Los empleados de consulta externa, le } \\
\text { dan una atención personal. }\end{array}$ & 0 & 0 & 0 & 0 & 0 & 0 & 0 & 0 & 3 & 3 & 36 & 47 & 37 & 49 & 76 \\
\hline $\begin{array}{l}\text { En consulta externa se preocupan } \\
\text { por sus mejores intereses. }\end{array}$ & 0 & 0 & 0 & 0 & 2 & 3 & 0 & 0 & 7 & 9 & 34 & 35 & 33 & 43 & 76 \\
\hline $\begin{array}{l}\text { Los empleados de consulta externa } \\
\text { comprenden sus necesidades específicas. }\end{array}$ & 0 & 0 & 0 & 0 & 0 & 0 & 0 & 0 & 2 & 3 & 32 & 42 & 42 & 55 & 76 \\
\hline
\end{tabular}

Fuente: datos obtenidos de la aplicación de la encuesta a la población objeto de estudio. Noviembre 2019. 
está parcialmente en desacuerdo. Por último, en el ítem 22 que evalúa si el profesional comprende las necesidades específicas del usuario, el 55\% está muy de acuerdo con la afirmación y el 3\% está parcialmente de acuerdo.

\section{DISCUSIÓN}

La temática relacionada con la calidad de los servicios de salud proporciona información precisa sobre como los usuarios perciben la atención; teniendo en cuenta el modelo SERVQUAL con sus cinco dimensiones, el cual permite comprender la importancia de la calidad en salud, desde la perspectiva del usuario mediante la satisfacción $(1,18)$.

De acuerdo con los resultados de la presente investigación, se pudo evidenciar que los usuarios que acudieron al servicio de consulta externa en el Centro de San Miguel del municipio de Tuta, perciben satisfacción al momento de recibir el servicio, desde las cinco dimensiones de elementos tangibles, fiabilidad, sensibilidad, seguridad y empatía; por tanto, según Niño y Perales (2), proponen que la diferencia entre las expectativas en los usuarios y sus percepciones en relación con el servicio recibido constituyen una medida de la calidad de servicio, enfocada en el área de salud; lo anterior, permite deducir que un alto porcentaje de satisfacción de los usuarios del Centro de Salud se determina por factores influyentes, tales como aspectos sociales, culturales y económicos, así mismo, el nivel de prestación de los servicios, el régimen especial de salud al que pertenecen, el respeto por el usuario al cumplir con el horario de atención y la disponibilidad del personal de salud en favor del usuario $(10,18)$.

La dimensión calificada con una puntuación más alta fue empatía, en esta línea, Infante Gómez (6), refiere que la empatía está directamente relacionada con el "trato con amabilidad, respeto y paciencia del personal de consulta externa"; de igual forma, se puede manifestar que la dimensión más cercana en el estudio de brechas fue empatía, concluyendo que los usuarios se encuentran satisfechos en esta dimensión, ya que la califican como un $73,7 \%$ de buena con mayor porcentaje $(83,1 \%)$ en los aspectos de trato con amabilidad, respeto y paciencia del personal de consulta externa. Así mismo, en un estudio realizado en un Hospital Universitario en Líbano, se halló que la brecha más baja fue para la empatía con un promedio de 0.5938 . En la misma revisión exploratoria, Polonia reporta que los aspectos que tienen mayor influencia en la percepción de la calidad son los costos, las condiciones de vida y otros asuntos relacionados con la empatía, la competencia del personal y su comunicación con el paciente, pero en especial, la satisfacción del paciente con su hospitalización $(6,19)$.

Por tanto, se puede concluir que los resultados de esta investigación que reportan satisfacción, coinciden con diferentes estudios de calidad de 
atención y grado de satisfacción de los usuarios de consulta externa de diferentes Centros de Atención de Salud, relacionándose posiblemente al trato que reciben los usuarios por parte del personal de salud $(6,18,20)$.

Continuando, las dimensiones fiabilidad y sensibilidad fueron las siguientes en obtener mayor calificación; en este sentido, se puede evidenciar cuánto influye la actitud del personal de salud presto a solucionar dudas y favorecer el servicio de los usuarios; de acuerdo con los resultados, los profesionales realizan bien el servicio a la primera, en el tiempo prometido e insisten en mantener registros exentos de errores; lo anterior, indica que el usuario de consulta está satisfecho con el trato recibido por parte del personal médico de esta institución, además, que la tendencia en general es hacia la satisfacción en cuanto a las variables que miden el recurso físico y humano de la institución (10,21-23).

La dimensión calificada con una puntuación más baja fue elementos tangibles, por ello, se puede precisar que las instalaciones no son del todo atractivas ni con apariencia limpia y agradable, así como tampoco los equipos están en excelentes condiciones. Cabe señalar que los elementos tangibles es lo que aprecia directamente una persona en cuanto a los aspectos físicos del servicio y son supremamente importantes para la calidad de atención, lo que por supuesto satisface al usuario, como lo refieren algunos estudios $(3,23)$. Por el contrario, los resultados de esta investigación que reportan menor satisfacción, no coinciden con el estudio de percepción de los usuarios frente a la calidad del servicio de salud en consulta externa en una Institución Prestadora de Servicios de Salud en Tunja Boyacá, ya que la dimensión con una calificación más alta fue elementos tangibles, esto quiere decir que los usuarios se encuentran satisfechos con las instalaciones, los equipos y el personal (11); de igual manera, tampoco coinciden con el estudio de Satisfacción en primer nivel de atención médica realizado en México, donde la satisfacción ante los elementos tangibles fue de un $82 \%$ (23). Todo lo anterior genera una importante recomendación a la institución frente a los elementos tangibles, ya que son representativos para los usuarios.

Finalmente, la dimensión de seguridad es categorizada como aceptable, lo que indica que el comportamiento del profesional de consulta externa, transmite algún grado de confianza, amabilidad y que tienen conocimientos para responder a las inquietudes del usuario. La seguridad del paciente es muy importante al momento de prestar un servicio de salud por cuanto se busca este se sienta tranquilo y seguro con respecto a los profesionales de salud y al ambiente institucional $(4,22)$. Tal como lo refieren Vitarte y Enrique, quienes sustentan que el instrumento SERVQUAL ayuda a los establecimientos de salud a 
evaluar hasta qué punto la cultura de seguridad de su organización transmite la importancia de la seguridad del paciente $(3,20)$.

Ante estos hallazgos concluyentes, se puede evidenciar que el servicio de consulta externa evaluado presta una buena atención a sus usuarios, la cual satisface sus necesidades, pero requiriendo siempre de estrategias de mejora en las que se pretenda satisfacer las necesidades y demandas de los usuarios, brindado calidad en la prestación de los servicios de salud. De igual forma, se recomienda que en futuras investigaciones se vincule la subjetividad de los usuarios $y$, de esta forma, brindar herramientas más específicas y oportunas en cuanto la planeación e implementación de estrategias

Como limitación del estudio, se evidencia la necesidad de una mirada cualitativa de la investigación y de los sujetos abordados, que favorezca la comunicación y exteriorización de necesidades particulares.

\section{AGRADECIMIENTO}

A los directivos de la E.S.E Centro de San Miguel del municipio de Tuta, por permitirnos la ejecución de la investigación en dicha institución. A nuestra asesora Marcela Arenas.

\section{CONFLICTO DE INTERÉS}

Los autores declaran no tener ningún conflicto de interés.

\section{FINANCIACIÓN}

Investigación financiada por las autoras.

\section{REFERENCIAS}

1. Ochante J, Hinojosa G, Ayala F, Pérez C. Satisfacción de la usuaria en consulta externa del servicio de ginecología en el Instituto Nacional Materno Perinatal. Revista Peruana de Investigación Materno Perinatal, 2015;4(1):32-37. https://doi. org/10.33421/inmp.21541

2. Effio B, Carrasco J, Chavesta M, Leguía X, Díaz J, Calidad de servicio en la consulta externa de un hospital de la región Lambayeque octubre 2010. Rev. Cuerpo Médico 2012;5(1):8-9. Available from: https://dialnet.unirioja.es/servlet/ articulo? codigo $=4061256$

3. Cobo-Mejía E, Estepa-Rodríguez K, Herrera-Triana C, Linares-Reyes P. Percepción de los usuarios frente a la calidad del servicio de salud en consulta externa en una Institución Prestadora de Senvicios de Salud. Revista Investigación en Salud Universidad de Boyacá. 2018;5(2):277-94. https:// doi.org/10.24267/23897325.321

4. Vásquez R, Amado J, Ramírez F, Velásquez R, Huari $R$. Sobredemanda de atención médica en el servicio de emergencia de adultos de un hospital terciario, Lima, Perú. An. Fac. med. [Internet]. 2016 [citado 2019 Ago.15]; 77(4):379-385. Available from: 
http://www.scielo.org.pe/scielo.php?script $=$ sci arttext\&pid=S1025-55832016000400010\&lng $=e$ s\&tlng=es

5. Freitas J, Silva A, Bauer C, Minamisava R, Bezerra A, Sousa M, Regina G. Quality of nursing care and satisfaction of patients attended at a teaching hospital. Rev. Latino-Am. Enfermagem. 2014;22(3):454-460. https://doi.org/10.1590/01041169.3241 .2437

6. Infantes F. Calidad de atención y grado de satisfacción de los usuarios de consulta externa del Centro de Atención de Medicina Complementaria del Hospital III Iquitos-2016. Revista Peruana de Medicina Integrativa. 2017; 2(2) 133-9. Available from: https://www.rpmi.pe/ojs/index.php/RPMI/ article/viewFile/55/54\#page $=1$

7. Hernández I, González T, Villanueva C, García S, Martín C, Ruiz, M. Arnau S. Revista de calidad asistencial, ISSN 1134-282X. 2010; 25(2) 83-89. Available from: https://dialnet.unirioja.es/servlet/ articulo? codigo $=3320464$

8. Díaz-Grajales C, Zapata-Bermúdez Y, AristizábalGrisales JC. Acceso y satisfacción con servicios curativos: Análisis de casos en afiliados al régimen contributivo y afiliados al régimen subsidiado en un barrio estrato 2 - Cali, Colombia. Rev Gerenc y Polit Salud. 2015;14(29):155-78. https://doi. org/10.11144/Javeriana.rgyps14-29.assc
9. Araujo R, Clemenza C, Fuenmayor R, Altuve E. Percepción de la calidad desenvicio de los usuarios de los programas sociales en salud. Revista de Ciencias Sociales [Internet]. 2010;16(1):80-91. Disponible en: http://www.scielo.org.ve/scielo.php?script=sci_ arttext\&pid =S1315-95182010000100008

10. Boada A, Barbosa A, Cobo E. Percepción de los usuarios frente a la calidad de atención en salud del servicio de consulta externa según el modelo SERVQUAL. Revista Investigación En Salud Universidad De Boyacá. 2019;6(1):55-71. https:// doi.org/10.24267/23897325.408

11. Baig MA, MianA, NajeedF, Shahzad H. Overcrowding in the emergency departments: Challenges and opportunities for improvement. J Pak Med Assoc. 2015;65(12):1344-5. Available from: http://jpma. org.pk/full_article_text.php?article_id $=7560$

12. Delgado ME, Vázquez ML, Moraes L. Calidad en los servicios de salud desde los marcos de sentido de diferentes actores sociales en Colombia y Brasil. Rev Salud Pública. 2011;12(4):533-45. https://doi. org/10.1590/S0124-00642010000400001

13. Morales MSL, León P, Trejo M del C. Calidad de la atención desde la perspectiva del usuario en una comunidad terapéutica en Veracruz, México. Revista Investigación en Salud Universidad de Boyacá. 2015;2(2):177-94. https://doi. org/10.24267/23897325.136 
14. González J, Díaz S, Hernández S, Huérfano S, Ibarra S, Jiménez A, Lara D, León I, Lizcano E. Satisfacción de pacientes que asisten a consulta médica ambulatoria en una EPS de régimen especial. Carta Comunitaria. 2018;23(134):4-14. https://doi. org/10.26752/ccomunitaria.v23.n134.262

15. Kerguelén C. Calidad en salud en Colombia. Ministerio de la Protección Social, Programa de Apoyo a la Reforma de la Salud - PARS. 2008. 233 p. Available from: https://www.minsalud. gov.co/Documentos\%20y\%20Publicaciones/ CALIDAD \%2 0EN\%20SALUD\% 20EN\%20 COLOMBIA.pdf

16. Nishizawa RM. Desarrollo del Modelo Servqual para la medición de la calidad del senvicio en la empresa de publicidad Ayuda Experto. Perspectivas [Internet]. 2014;(34):181-209. Available from: http://www.redalyc.org/pdf/4259/425941264005. pdf

17. Numpaque $A$, Rocha $A$. Modelos SERVQUAL y SERVQHOS para la evaluación de calidad de los servicios de salud. Rev la Fac Med. 2016;64(4):715.

18. Niño B, Perales J, Chavesta X, Leguía J, Díaz C. Calidad de Servicio en la consulta externa de un hospital de la región Lambayeque octubre 2010. Rev cuerpo méd HNAAA [Internet]. 2012;5(1):5-9. Available from: https://dialnet.unirioja.es/servlet/ articulo? codigo $=4061256$
19. Yépez-Chamorro MC, Ricaurte-Cepeda M, Jurado DM. Calidad percibida de la atención en salud en una red pública del municipio de Pasto, Colombia. Univ y Salud. 2018;20(2):97.

20. Rodríguez $M$, Muñoz R. Factores relacionados con la satisfacción en consultas externas en Hospitales de Madrid. Rev Ekectronica Enferm Actual en Costa Rica [Internet]. 2017;32:1-13. Available from: https://dialnet.unirioja.es/servlet/ articulo? codigo $=5774441$

21. Al-Momani MM. Gap analysis between perceptions and expec- tations of medical-surgical patients in a public hospital in Saudi Arabia. Med Princ Pract 2015;25(1):79-84. https://doi. org/10.1159/000441000

22. Barragán JA, Moreno C. Calidad percibida por usuarios de enfermería en tres hospitales públicos. Enfermería Global. 2013;12(1):217-30.

23. Vázquez-Cruz E, Sotomayor-Tapia J, GonzálezLópez A, Montiel-Jarquín A, Gutiérrez-Gabriel I, Romero-Figueroa MS, et al. Satisfacción del paciente en el primer nivel de atención médica. Rev Salud Pública. 2018;20(2):254-7.

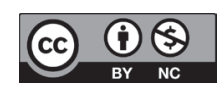

Esta obra está bajo una licencia de Creative Commons Reconocimiento-NoComercial 4.0 Internacional 